\title{
COMPUTABLE ANALYSIS AND BLASCHKE PRODUCTS
}

\author{
ALEC MATHESON AND TIMOTHY H. MCNICHOLL \\ (Communicated by Julia Knight) \\ Dedicated to the memory of Alec Matheson.
}

\begin{abstract}
We show that if a Blaschke product defines a computable function, then it has a computable sequence of zeros in which the number of times each zero is repeated is its multiplicity. We then show that the converse is not true. We finally show that every computable, radial, interpolating sequence yields a computable Blaschke product.
\end{abstract}

\section{INTRODUCTION}

A primary goal of computable analysis is to examine the methods of analysis for their constructive content. An analyst desiring a constructive proof of some theorem for the sake of future development may be pointed in the direction of a constructive proof by computable analysis, or may be led to conclude that no such proof is possible. In addition, computable analysis provides a reasonable idealization of the task of programming numerical solutions to problems from analysis so that the limits of this activity can be explored.

To date, most of the research in computable analysis has been on real analysis, and much of it has been foundational. Here, we focus on complex analysis. In particular, we focus on topics in the theory of bounded analytic functions. This is a rich theory that has undergone significant development in the past half-century. We use recent advances in the foundations of computable analysis to initiate the examination of this theory for its constructive content. We find that in this area there are many opportunities to exploit beautiful interactions between ideas of computability and classical analysis. We hope to continue this examination in the future, and we also hope that researchers in both complex analysis and computability will do the same.

We note that this is not the first paper to address issues in complex analysis from the perspective of computable analysis. See, for example, [2] and [5].

Let us now be more specific. Let $\mathbb{D}$ be the open unit disk centered at the origin. An inner function is a bounded analytic function from $\mathbb{D}$ into $\mathbb{C}$ whose modulus approaches 1 at almost all points on $\partial \mathbb{D}$. Our goal is to study the space of inner functions from the perspective of computable analysis. We use Type Two Effectivity Theory as developed in [13] as our foundation. We find however, that learning the setup of this theory involves absorbing a fair amount of technical detail. However,

Received by the editors June 2, 2006 and, in revised form, January 27, 2007.

2000 Mathematics Subject Classification. Primary 03F60, 30D50.

(C) 2007 American Mathematical Society 
in the context of the unit disk, there are some equivalents to the fundamental notions of this theory (e.g. computable number and computable function) which are less technical. We will state and prove these equivalents. We also use Church's Thesis (see [11]) rather than explicitly constructing Turing machines to carry out computations on the natural numbers.

We now turn to an important kind of inner function. A Blaschke product is a product of the form

$$
z^{m} \prod_{n=0}^{\infty} \frac{\left|a_{n}\right|}{a_{n}} \frac{a_{n}-z}{1-\overline{a_{n}} z} .
$$

If the Blaschke condition $\sum_{n=0}^{\infty}\left(1-\left|a_{n}\right|\right)<\infty$ is met, then the zeros of this function are precisely the terms of the sequence $\left\{a_{n}\right\}_{n=0}^{\infty}$, and the number of times a zero is repeated in this sequence is its multiplicity. If this condition is not met, then the product is zero for all values of $z$. The poles of this function all lie outside $\mathbb{D}$. For a thorough introduction to Blaschke products, see Chapter 15 of [10] and Chapter II, Section 2 of [4]. Inner functions and Blaschke products continue to be active areas of research. For example, see [6] and [9].

Since the set of Blaschke products is uniformly dense in the space of inner functions, we focus on Blaschke products. We prove two foundational theorems. Namely, that if a Blaschke product defines a computable function, then it has a computable sequence of zeros in which each zero is repeated according to its multiplicity. (In the next section, we discuss the precise meaning of the term computable when applied to complex functions and sequences of complex numbers.) We then show that the converse does not hold.

We also show that if $\left\{a_{n}\right\}_{n=0}^{\infty}$ is a computable Blaschke sequence, and if $\sum_{n=0}^{\infty}\left(1-\left|a_{n}\right|\right)$ is a computable real number, then the associated Blaschke product is a computable function. As a corollary, we show that a computable Blaschke sequence (i.e. a sequence satisfying the Blaschke condition) defines a computable function if the limit supremum in the ratio test is less than one when applied to the Blaschke sum $\sum_{n=0}^{\infty}\left(1-\left|a_{n}\right|\right)$. As an additional corollary, we obtain that every radial computable interpolating sequence defines a computable Blaschke product.

\section{Prerequisites From COMPUtability and COMPUtable ANALysis}

Unless otherwise mentioned, all computable analysis notation, in particular that of Type Two Effectivity, is as in Weihrauch [13].

Notation 2.1. We write $f: \subseteq A \rightarrow B$ if $\operatorname{dom}(f) \subseteq A$ and $\operatorname{ran}(f) \subseteq B$.

Definition 2.2. A rational rectangle is a set of the form $(a, b) \times(c, d)$ where $a, b, c, d$ are rational numbers, $a<b$, and $c<d$.

We note that the intersection of two rational rectangles is either a rational rectangle or is empty. If $R$ is a rational rectangle, then let $\operatorname{diam}(R)$ be the diameter of $R$.

A rational rectangle can be identified with an quadruple of rational numbers. It is well-known that each pair of natural numbers can be identified with a natural number in a computable fashion. It is also well-known that each integer can be computably represented by a pair of natural numbers. It follows that we can computably represent rational rectangles by natural numbers. We let $R_{n}$ denote the $n$th such rectangle. We call $n$ a code of $R_{n}$. 
A set of natural numbers $A$ is computable if there is an algorithm that given any $m \in \mathbb{N}$ as input determines if $m \in A$.

Convention 2.3. Unless otherwise mentioned, the following conventions regarding the notation of Type Two Effectivity are followed for the sake of brevity.

(1) A complex number $z$ is computable if it is $\rho^{2}$-computable, or equivalently, if there is an algorithm that given any $k \in \mathbb{N}$ as input computes a code of a rational rectangle that contains $z$ and whose diameter is at most $\frac{1}{2^{k}}$.

(2) A sequence $\left\{z_{n}\right\}_{n=0}^{\infty}$ of complex numbers is computable if it is $\left[\rho^{2}\right]^{\omega}$-computable, or equivalently, if there is an algorithm that given $n, k \in \mathbb{N}$ as input produces a code of a rational rectangle that contains $z_{n}$ and whose diameter is at most $\frac{1}{2^{k}}$.

(3) On the other hand, if the sequence $\left\{a_{n}\right\}_{n=0}^{\infty}$ is stipulated to contain only rational numbers, then we will say it is computable just in case there is an algorithm that given $n \in \mathbb{N}$ as input produces $m, d \in \mathbb{Z}$ such that $a_{n}=m / d$.

(4) A function $f: \subseteq \mathbb{C} \rightarrow \mathbb{C}$ is computable if it is $\left(\rho^{2}, \rho^{2}\right)$-computable. (We give an equivalent below in Theorem 2.7.)

A function $F: \subseteq \mathbb{N} \rightarrow \mathbb{N}$ is computable if there is an algorithm that given any $m \in \mathbb{N}$ as input outputs $F(m)$ if $m \in \operatorname{dom}(F)$ and does not halt if $m \notin \operatorname{dom}(F)$.

Suppose $F: \subseteq \mathbb{N} \rightarrow \mathbb{N}$ and $f: \subseteq \mathbb{C} \rightarrow \mathbb{C}$. We say that $F$ induces $f$ if for every $z \in \operatorname{dom}(f), f(z)$ is the only element of

$$
\bigcap\left\{R_{F(n)} \mid n \in \operatorname{dom}(F) \wedge z \in R_{n}\right\} .
$$

We will need the following three propositions. The first is an easy consequence of Corollary 3.2.12 of [13]. The second is an easy consequence of Corollary 6.2.5 of [13]. The third is an easy consequence of Lemma 4.1.19 of [13].

Proposition 2.4. Suppose $D$ is an open disk centered at the origin with rational radius. If $f: D \rightarrow \mathbb{C}$ is computable, then it is continuous.

Proposition 2.5. Suppose $D$ is an open disk centered at the origin with rational radius. Suppose $f: D \rightarrow \mathbb{R}$ is computable. Then, there is an algorithm that, given $n, k$ as input, produces rational numbers $r_{0}, r_{1}$ such that $r_{0}<\max f\left[\overline{R_{n}}\right]<r_{1}$ and $r_{1}-r_{0}<\frac{1}{2^{k}}$, provided $\overline{R_{n}} \subseteq D$.

Proposition 2.6. Suppose $D$ is an open disk centered at the origin with rational radius. If $f: D \rightarrow \mathbb{C}$ is computable, then $\operatorname{Re}(f)$ and $\operatorname{Im}(f)$ are computable.

The following theorem gives an equivalent condition to the meaning of ' $f: \subseteq$ $\mathbb{C} \rightarrow \mathbb{C}$ is computable' when the domain of $f$ is a disk centered at the origin and with rational radius. The proof is included for the sake of completeness. The reader willing to trust this equivalent and use it as his definition may skip the proof and move on to the main thrust of the paper.

Theorem 2.7. Let $D$ be a disk centered at the origin and with rational radius. A function $f: D \rightarrow \mathbb{C}$ is computable if and only if it is induced by a computable $F: \subseteq \mathbb{N} \rightarrow \mathbb{N}$. Furthermore, we can choose $F$ so that it has the following two properties.

- For every $z \in \operatorname{dom}(f)$ and every $\epsilon>0$, there exists $n \in \operatorname{dom}(F)$ such that $z \in R_{n}$ and $\operatorname{diam}\left(R_{n}\right)<\epsilon$.

- Whenever $m, n \in \operatorname{dom}(F)$ and $R_{n} \supseteq R_{m}, R_{F(n)} \supseteq R_{F(m)}$. 
Proof. Let $D$ be a disk centered at the origin and with rational radius.

$(\Rightarrow)$ : Suppose $f: D \rightarrow \mathbb{C}$ is computable. We construct a function $F: \subseteq \mathbb{N} \rightarrow \mathbb{N}$ having the required properties. We first introduce some notation.

By Proposition 2.6, $\operatorname{Re}(f)$ and $\operatorname{Im}(f)$ are computable. Hence, by Proposition 2.5, $R e(f)$ and $\operatorname{Im}(f)$ are continuous. When $\overline{R_{n}} \subseteq D$, let $M_{0}(n), M_{1}(n)$ be the maximum on $\overline{R_{n}}$ of $\operatorname{Re}(f)$ and $\operatorname{Im}(f)$, respectively; let $m_{0}(n), m_{1}(n)$ be their minima on $\overline{R_{n}}$.

We build $F$ by stages as follows.

Stage $t$ : Search for $n_{t, 1}, \ldots, n_{t, k_{t}}$ such that $\frac{1}{2^{t+1}}<\operatorname{diam}\left(R_{n_{t, i}}\right)<\frac{1}{2^{t}}$ for all $1 \leq i \leq k_{t}$ and $R_{n_{t, 0}}, \ldots, R_{n_{t, k_{t}}}$ form a minimal cover of $D$. That is, none of these rectangles is contained in another of these rectangles. If $\overline{R_{n_{t, i}}} \nsubseteq D$, then we declare $F\left(n_{t, i}\right)$ to be undefined. Suppose $\overline{R_{n_{t, i}}} \subseteq D$. By Proposition 2.5, we can compute rational numbers $m_{0}^{\prime}\left(n_{t, i}\right), m_{1}^{\prime}\left(n_{t, i}\right)$ such that $m_{j}^{\prime}\left(n_{t, i}\right)<m_{j}\left(n_{t, i}\right)$ and $\frac{1}{2^{t+1}}<m_{j}\left(n_{t, i}\right)-m_{j}^{\prime}\left(n_{t, i}\right)<\frac{1}{2^{t}}$ for each $j \in\{0,1\}$. It also follows that we can compute rational numbers $M_{0}^{\prime}\left(n_{t, i}\right), M_{1}^{\prime}\left(n_{t, i}\right)$ such that $M_{j}^{\prime}\left(n_{t, i}\right)<M_{j}\left(n_{t, i}\right)$ and $\frac{1}{2^{t}}<M_{j}\left(n_{t, i}\right)-M_{j}^{\prime}\left(n_{t, i}\right)<\frac{1}{2^{t+1}}$ for each $j \in\{0,1\}$. Define $F\left(n_{t, i}\right)$ to be a code of $\left(m_{0}^{\prime}\left(n_{t, i}\right), M_{0}^{\prime}\left(n_{t, i}\right)\right) \times\left(m_{1}^{\prime}\left(n_{t, i}\right), M_{1}^{\prime}\left(n_{t, i}\right)\right)$.

We now show that $F$ has the desired properties. We first show that if $z \in D$, then for every $\epsilon>0$ there exists $n \in \operatorname{dom}(F)$ such that $\operatorname{diam}\left(R_{n}\right)<\epsilon$. Let $z \in D$, and let $\epsilon>0$. Suppose $t$ is such that $\frac{1}{2^{t}}<\epsilon$. There exists $1 \leq i \leq k_{t}$ such that $z \in R_{n_{t, i}}$. However, it may be that $\overline{R_{n_{t, i}}}$ is not contained in $D$ for any such $i$. If this is the case for every (or even infinitely many) $t$ for which $\frac{1}{2^{t}}<\epsilon$, then we are led to conclude that $z$ is arbitrarily close to the boundary of $D$, and hence is on the boundary of $D$, which is not the case. Thus, there exists $n \in \operatorname{dom}(F)$ such that $\operatorname{diam}\left(R_{n}\right)<\epsilon$ and $z \in R_{n}$.

We now show that $R_{F(n)} \supseteq R_{F(m)}$ whenever $m, n \in \operatorname{dom}(F)$ and $R_{n} \supseteq R_{m}$. Suppose $m, n \in \operatorname{dom}(F)$ and $R_{n} \supseteq R_{m}$. Without loss of generality, suppose $R_{n} \supset$ $R_{m}$. There exist unique $i_{0}, t_{0}, i_{1}, t_{1}$ such that $n=n_{t_{0}, i_{0}}$ and $m=n_{t_{1}, i_{1}}$. Since $R_{m} \subset R_{n}$, it follows that $t_{1}>t_{0}$. Let $T_{m}$ denote

$$
\left[m_{0}\left(n_{t_{1}, i_{1}}\right), M_{0}\left(n_{t_{1}, i_{1}}\right)\right] \times\left[m_{1}\left(n_{t_{1}, i_{1}}\right), M_{1}\left(n_{t_{1}, i_{1}}\right)\right],
$$

and let $T_{n}$ denote

$$
\left[m_{0}\left(n_{t_{0}, i_{0}}\right), M_{0}\left(n_{t_{0}, i_{0}}\right)\right] \times\left[m_{1}\left(n_{t_{0}, i_{0}}\right), M_{1}\left(n_{t_{0}, i_{0}}\right)\right] .
$$

It follows that $T_{n} \supseteq T_{m}$. The rectangle $R_{F(m)}$ is obtained by slightly increasing the coordinates of the vertices of $T_{m}$, and the rectangle $R_{F(n)}$ is obtained by slightly increasing the coordinates of the vertices of $T_{n}$. By the construction of $F$, there is a number $l$ such that the amount the coordinates of $T_{m}$ are increased is less than $l$, and the amount the coordinates of $T_{n}$ are increased is larger than $l$. It follows that $R_{F(m)} \subseteq R_{F(n)}$.

We now turn to showing that $F$ induces $f$. Fix $z \in D$. It follows from the definition of $F$ that $f(z) \in R_{F(n)}$ whenever $z \in R_{n}$ and $n \in \operatorname{dom}(F)$. Hence,

$$
f(z) \in \bigcap\left\{R_{F(n)} \mid n \in \operatorname{dom}(F) \wedge z \in R_{n}\right\} .
$$

We now show that $f(z)$ is the only element of this intersection. To do so, it suffices to show that for every $\epsilon>0$ there exists $n \in \operatorname{dom}(F)$ such that $z \in R_{n}$ and $\operatorname{diam}\left(R_{F(n)}\right)<\epsilon$. As shown above, there exists $t_{0}$ such that for all $t \geq t_{0}$ there 
exists $i_{t}$ such that $z \in R_{n_{t, i_{t}}}$. For each $t \geq t_{0}$, let $T_{t}$ denote $\left(m_{0}\left(n_{t, i_{t}}\right), M_{0}\left(n_{t, i_{t}}\right)\right) \times$ $\left(m_{1}\left(n_{t, i_{t}}\right), M_{1}\left(n_{t, i_{t}}\right)\right)$. Then, $f(z) \in T_{t} \subseteq R_{F\left(n_{t, i_{t}}\right)}$ for all $t \geq t_{0}$. Again, the rectangle $R_{F\left(n_{t, i_{t}}\right)}$ is obtained by slightly increasing the coordinates of $T_{t}$. The amount of this increase tends to 0 as $t$ approaches infinity. By Proposition 2.4, $f$ is continuous. It then follows that the diameter of $T_{t}$ approaches 0 as $t$ approaches infinity, and that for every $\epsilon>0$ there exists $n \in \operatorname{dom}(F)$ such that $z \in R_{n}$ and $\operatorname{diam}\left(R_{F(n)}\right)<\epsilon$.

We have now shown that $F$ has all the required properties.

$(\Leftarrow)$ : Let $\rho^{b}$ be as in Lemma 4.1.6 of [13]. Suppose that $f: D \rightarrow \mathbb{C}$ is induced by $F: \subseteq \mathbb{N} \rightarrow \mathbb{N}$ and that $F$ is computable. It then follows that $f$ is $\left(\rho^{2},\left(\rho^{b}\right)^{2}\right)$ computable. (Informally speaking, as we descend down a $\rho^{2}$-name for $z, p$, we use $F$ to map the rectangles encountered to other rectangles which then allow us to form a $\left(\rho^{b}\right)^{2}$-name for $f(z)$.) Since $\rho^{b} \equiv \rho, f$ is $\left(\rho^{2}, \rho^{2}\right)$-computable.

We note that there are some similarities between Theorem 2.7 and the approach to computable analysis developed by Kalantari and Welch. Specifically, the forward direction is very similar to Theorem 5.3 of [7]. The other direction is very similar to Theorem 4.1 of [7]. A thorough discussion of the relationship between Kalantari and Welch's approach to computable analysis and Type Two Effectivity is beyond the scope of this paper. However, we mention in passing that the essential difference is that Type Two Effectivity provides a general approach for names of computable objects, while Kalantari and Welch's approach deals with a specific system of names. A thorough discussion of this other approach to computable analysis can be found in $[8]$.

We note that the proof of Theorem 2.7 shows that every continuous function $f: D \rightarrow \mathbb{C}$ is induced by a (not necessarily computable) function $F: \subseteq \mathbb{N} \rightarrow \mathbb{N}$. Furthermore, the proof of this theorem is uniform in that it effectively maps a function $F$ that induces $f$ to a $[\rho \rightarrow \rho]$-name of $f$ as in [13] and vice versa. The details of this are, however, beyond what we wish to consider in this paper, though they may have application to future investigations.

A set of natural numbers $A$ is computably enumerable if it is the range of a computable $F: \subseteq \mathbb{N} \rightarrow \mathbb{N}$, or equivalently, if either $A$ is empty or there is an algorithm that lists the elements of $A$. Every computable set is computably enumerable, but Turing's halting set provides a counterexample to the converse (see [11]).

Definition 2.8. Let $X \subseteq \mathbb{C}$.

(1) If $X$ is open, then we say that $X$ is computably enumerable if $\left\{n \mid \overline{R_{n}} \subseteq X\right\}$ is computably enumerable.

(2) If $X$ is closed, then we say that $X$ is computably enumerable if $\left\{n \mid R_{n} \cap X \neq\right.$ $\emptyset\}$ is computably enumerable.

We abbreviate computably enumerable by c.e.

\section{Basic COMPUtability ISSUes For BlaschKe PRODUCTS}

Notation 3.1. If $A=\left\{a_{n}\right\}_{n=0}^{\infty}$ is a sequence of complex numbers in the unit disk, then

$$
B_{A, m}(z)=_{d f} z^{m} \prod_{n=0}^{\infty} \frac{\left|a_{n}\right|}{a_{n}} \frac{a_{n}-z}{1-\overline{a_{n}} z} .
$$

Let $B_{A}=B_{A, 0}$. 
Definition 3.2. A Blaschke product $B$ is computably presented if there exists computable $A$ such that $B=B_{A, m}$ for some $m$.

Our first goal is to show that every computable Blaschke product is computably presented. We will need the following lemma.

Lemma 3.3. Let $U \subseteq \mathbb{C}$ be open and c.e. If $X \subseteq U$ is infinite, discrete, and closed relative to $U$, and if $\bar{X}$ is c.e., then there exists computable $\left\{a_{n}\right\}_{n=0}^{\infty}$ such that $X=\left\{a_{0}, a_{1}, \ldots,\right\}$ and $a_{i} \neq a_{j}$ for $i \neq j$.

Proof. We define an array of rational rectangles $\left\{R_{m, n}\right\}$. At every stage of the construction, $R_{m, n}$ is defined for only finitely many $m, n$. However, if $R_{m, n}$ is defined at a stage, then $R_{m, n^{\prime}}$ will be defined as well for all $n^{\prime}<n$ and will contain $R_{m, n}$. Write $R_{m, n}[t] \downarrow$ if $R_{m, n}$ is defined by the end of stage $t$, and write $R_{m, n}[t] \uparrow$ otherwise.

Begin by enumerating all rational rectangles that intersect $X$ and whose closures are contained in $U$. Let $R_{0,0}$ be the first rectangle enumerated.

Suppose $R$ is enumerated at stage $t+1$. If for every $m$ such that $R_{m, 0}[t] \downarrow$ there exists $n$ such that $R_{m, n}[t] \downarrow$ and $R_{m, n} \cap R=\emptyset$, then let $R_{k, 0}=R$ where $k$ is the least number such that $R_{k, 0}[t] \uparrow$. Suppose there exists $m$ such that $R_{m, 0}[t] \downarrow$ and $R_{m, n} \cap R \neq \emptyset$ whenever $R_{m, n}[t] \downarrow$. If there exists $m$ such that $R_{m, n} \supset R$ and the diameter of $R$ is at most $\frac{1}{2^{n+1}}$ where $n$ is the largest number such that $R_{m, n}[t] \downarrow$, then let $R_{m, n+1}=R$. (By way of induction, such an $m$ is unique.) If no such $m$ is found, then we go on to the next stage without doing anything.

Since $X$ is infinite, it follows that $R_{m, n}$ is defined for all $m, n$. Since the diameter of $R_{m, n+1}$ is at most $\frac{1}{2^{n+1}}$ for all $n$, it follows that $\bigcap_{n} R_{m, n}$ contains exactly one point. For each $m$, define $a_{m}$ to be this point. Since $X$ is closed relative to $U, a_{m} \in X$ for all $m$. Clearly, $\left\{a_{m}\right\}_{m=0}^{\infty}$ is computable. By way of contradiction, suppose $z \in X$ and $z \neq a_{m}$ for all $m$. There are arbitrarily small rational rectangles containing $z$ and no other point of $X$ and whose closures are contained in $U$. Hence, whenever such a rectangle is enumerated, it must intersect every rectangle in a partially defined row of $\left\{R_{m, n}\right\}_{m, n}$. It follows that there are points of $X$ arbitrarily close to $z$; a contradiction since $X$ is discrete.

We note that the proof of Lemma 3.3 is uniform in that it yields an algorithm which, when applied to an algorithm for enumerating

$$
\left\{n \mid R_{n} \cap \bar{X} \neq \emptyset\right\}
$$

and an algorithm for enumerating

$$
\left\{n \mid \overline{R_{n}} \subseteq U\right\},
$$

yields an algorithm which computes $\left\{a_{n}\right\}_{n=0}^{\infty}$ (in the sense of Convention 2.3 part $(2))$.

Theorem 3.4. If $U \subseteq \mathbb{C}$ is open and c.e., and if $f: U \rightarrow \mathbb{C}$ is computable, not identically zero, and analytic, then $f^{-1}[\{0\}]$ is discrete and its closure is c.e.

Proof. Since $f$ is analytic and not identically zero, $f^{-1}[\{0\}]$ is discrete. It follows from the Cauchy integral formula and Theorem 6.4.1 of [13] that $f^{\prime}$ is computable.

Begin enumerating all open rational rectangles $R$ for which $\bar{R} \subseteq U$. For each such rectangle $R$, begin simultaneously estimating the value of

$$
\frac{1}{2 \pi i} \int_{\partial R} \frac{f^{\prime}}{f}
$$


If there are no zeros of $f$ on $\partial R$, then this integral is of course defined. If there is a zero of $f$ on $\partial R$, then this integral may not be defined, but we can still apply this estimation procedure. In this case, the results, if any, will of course be meaningless. However, we will later show that such results, or absence of results, will not adversely affect the final output of the enumeration procedure we are defining. For future reference, let us call (3.1) a garbage integral when $\partial R$ contains a zero of $f$.

The key claim is that whenever the value of (3.1) is located within a rational open interval containing exactly one positive integer, we know that $\bar{R}$ contains a zero of $f$. For, let us suppose that for some $R$, we have at some point in time through our estimation procedure located the value of (3.1) inside such an interval. Now, if (3.1) is not a garbage integral, then by the Argument Principle $R$, and hence $\bar{R}$, contains a zero of $f$. However, if (3.1) is a garbage integral, then $\partial R$, and hence $\bar{R}$, contains a zero of $f$. So, whether (3.1) is a garbage integral or not, whenever our estimating procedure locates the value of this integral inside a rational open interval containing exactly one positive integer, we can be assured that $\bar{R}$ contains a zero of $f$ (though $R$ may not).

Hence, whenever, for some $R$, we so locate the value of (3.1), we enumerate every rational rectangle that contains $\bar{R}$. All of these rectangles must contain a zero of $f$ since $\bar{R}$ does.

Now, this procedure ensures that every rectangle enumerated contains a zero of $f$. But, to complete the proof, we must show that every rectangle that contains a zero of $f$ is enumerated at some point. Suppose $S$ is a rational rectangle that contains a zero of $f$. Since the zeros of $f$ are isolated, there is a rational rectangle $S^{\prime}$ such that $\overline{S^{\prime}} \subseteq S \cap U, S^{\prime}$ contains a zero of $f$, and $\partial S^{\prime}$ does not contain a zero of $f$. The above procedure will eventually encounter $S^{\prime}$, and at some point in time will locate the value of the corresponding integral in a rational open interval containing exactly one positive integer. At some point in time afterwards, the rectangle $S$ will be enumerated.

It follows that the closure of $f^{-1}[\{0\}]$ is c.e. ${ }^{1}$

We remark that the above proof is uniform in that there is an algorithm that given two algorithms as input, one of which computes a function that induces $F$, and one of which enumerates

$$
\left\{n \mid \overline{R_{n}} \subseteq U\right\},
$$

produces an algorithm that enumerates all rational rectangles which contain a zero of $f$.

The following notation will prove useful.

Notation 3.5. $b_{a}(z)=\frac{|a|}{a} \frac{a-z}{1-\bar{a} z}$.

We now have everything we need to prove the main theorem of this section.

\footnotetext{
${ }^{1}$ One can modify this procedure so as to remove its "garbage in, garbage out" nature. For, it follows from Theorem 6.2 .5 of [13] that $n \mapsto \min \left\{|f(z)|: z \in \partial R_{n}\right\}$ is computable. Now, we cannot test whether this minimum is zero, but we can enumerate the values of $n$ for which it is greater than zero. So, at the beginning of the procedure in this proof, rather than enumerating all rational rectangles contained in $U$, we could enumerate all rational rectangles contained in $U$ which do not have a zero of $f$ on their boundary while at the same time estimating the values of the corresponding integrals. We have included this modified procedure since we believe both lines of reasoning may prove valuable to future research. We thank the referee for pointing out this modification.
} 
Theorem 3.6. Every computable Blaschke product is computably presented.

Proof. Suppose $B$ is a computable Blaschke product. Without loss of generality, we may assume that $B$ is not identically zero. Thus, the Blaschke sum of $B$ has finite value. Hence, there are infinitely many zeros of $B$.

By Theorem 3.4, $B^{-1}[\{0\}]$ is c.e. We now form a sequence $A=\left\{a_{n}\right\}_{n=0}^{\infty}$ as follows. Use the procedure in the proof of Lemma 3.3 to begin forming a computable sequence $\left\{a_{n, 0}\right\}_{n=0}^{\infty}$ whose terms are precisely the elements of $B^{-1}[\{0\}]$. Set $a_{0}=$ $a_{0,0}$.

The function $B / b_{a_{0}}$ has a singularity only at $a_{0}$, and this singularity is removable. If we let $B_{1}$ be the function that results, then we obtain another Blaschke product. We now want to apply the procedure of Theorem 3.4 to $B_{1}$. The only obstacle is the singularity at $a_{0}$. However, using the fact that $a_{0}$ is computable, we can in that procedure use only rectangles $R$ such that $a_{0} \notin \partial R$ and get the same result. Furthermore, $B$, when considered as a function from $\mathbb{D}-\left\{a_{0}\right\}$ into $\mathbb{D}$, is analytic and computable and so has a computable derivative.

So we can simultaneously form a computable sequence $\left\{a_{n, 1}\right\}_{n=0}^{\infty}$ whose terms are precisely the elements of $B_{1}^{-1}[\{0\}]$. Set $a_{1}=a_{0,1}$. Set $B_{2}=B_{1} / b_{a_{1}}$ (i.e. the function that results from removing the singularity at $a_{1}$ of $\left.B_{1} / b_{a_{1}}\right)$. Repeat this procedure to obtain $a_{2}, a_{3}, \ldots$. Clearly, the terms of $\left\{a_{n}\right\}_{n=0}^{\infty}$ are precisely the zeros of $B$, and the number of times each zero appears in this sequence is its multiplicity. It follows that $B=B_{A}$.

We mention here that the proof of Theorem 3.6 can be extended to yield a much stronger result. First of all, let us say that a function $F: \mathbb{N} \rightarrow \mathbb{N}$ induces a sequence $\left\{a_{n}\right\}_{n=0}^{\infty}$ if for each $(n, k), a_{n} \in R_{F(n, k)}$ and $\operatorname{diam}\left(R_{F(n, k)}\right)<\frac{1}{2^{k}}$. (Hence, the computable sequences are precisely those induced by computable functions.) The above proof yields an "algorithm" which transforms a function that induces a not identically zero Blaschke product (which need not be computable) into a function that induces its sequence of zeros. The details involved in precisely stating and proving this result are beyond the scope of this paper. These can be found in Chapter 6 of [13] which deals with computing on function spaces.

We now turn to showing that not every computably presented Blaschke product is computable.

Theorem 3.7 (Specker [12]). There exists a computable sequence of rational numbers $1>r_{0}>r_{1}>\ldots>0$ such that $\lim _{n \rightarrow \infty} r_{n}$ is not computable.

Lemma 3.8. If $f: \mathbb{D} \rightarrow \mathbb{C}$ is computable, then $f(z)$ is computable for every computable $z \in \mathbb{D}$.

Proof. This follows from Theorem 2.1.13 of [13]. For the sake of completeness, we give a proof here based only on the equivalent given by Theorem 2.7.

Let $F: \subseteq \mathbb{N} \rightarrow \mathbb{N}$ be a computable function that induces $f$ and that satisfies the conditions of Theorem 2.7. Let $z \in \operatorname{dom}(f)$ be computable. It follows that $f(z)$ is the unique element of

$$
\bigcap\left\{R_{F(n)} \mid z \in R_{n} \wedge n \in \operatorname{dom}(F)\right\} .
$$

Let $m \in \mathbb{N}$ be given as input. Search for $n_{1}, \ldots, n_{k} \in \operatorname{dom}(F)$ such that the diameter of $\bigcap_{j=1}^{k} R_{F\left(n_{j}\right)}$ is at most $\frac{1}{2^{m}}$ and each $R_{n_{j}}$ contains a rational rectangle 
that contains $z$. (The latter search condition is made possible by the assumption that $z$ is computable.) Output a code of this rectangle.

Theorem 3.9. Suppose $z_{0} \in \mathbb{D}$ is real and positive. There is a computable sequence of points in $\mathbb{D}, A=\left\{a_{n}\right\}_{n=0}^{\infty}$, such that the following hold.

(1) A satisfies the Blaschke condition.

(2) $B_{A}\left(z_{0}\right)$ is real and not computable. Hence, if $z_{0}$ is computable, then $B_{A}$ is not computable.

Proof. Let $r_{n}$ be as in Theorem 3.7. Let $c=\lim _{n \rightarrow \infty} r_{n}$. Define:

$$
\begin{gathered}
c_{0}=r_{0}, \\
c_{n+1}=\frac{r_{n+1}}{r_{n}}, \\
a_{n}=\frac{c_{n}+z_{0}}{1+c_{n} z_{0}} .
\end{gathered}
$$

Since $\left\{r_{n}\right\}_{n=0}^{\infty}$ is decreasing, $0<c_{n}<1$ for all $n$. Each $a_{n}$ is obtained by applying the Möbius transformation $z \mapsto \frac{z+c_{n}}{1+c_{n} z}$ to $z_{0}$. Each of these Möbius transformations is an automorphism of $\mathbb{D}$. Hence, $a_{n} \in \mathbb{D}$. Furthermore, by a simple calculation

$$
B_{A}\left(z_{0}\right)=\prod_{n=0}^{\infty} c_{n}=c .
$$

By Lemma 3.9, $B_{A}$ is not computable. Since $B_{A}\left(z_{0}\right) \neq 0$, it follows that $\sum_{n=0}^{\infty}\left(1-\left|a_{n}\right|\right)<\infty$.

Corollary 3.10. Not every computably presented Blaschke product is computable.

\section{A connection with the ratio test \\ AND WITH INTERPOLATING SEQUENCES}

Notation 4.1. For each $k \in \mathbb{N}$, let $\mathbb{D}_{k}=\left\{z \in \mathbb{D}|| z \mid<1-\frac{1}{2^{k+1}}\right\}$.

Definition 4.2. Suppose $F_{k}: \subseteq \mathbb{N} \rightarrow \mathbb{N}$ for all $k$. We say that the sequence of functions $\left\{F_{k}\right\}_{k \in \mathbb{N}}$ is uniformly computable if there exists an algorithm that given $k, n$ as input outputs $F_{k}(n)$ if $n \in \operatorname{dom}\left(F_{k}\right)$ and does not halt otherwise.

Lemma 4.3. Suppose $f: \mathbb{D} \rightarrow \mathbb{D}$ and for each $k \in \mathbb{N}$ the restriction of $f$ to $\mathbb{D}_{k}$ is induced by a map $F_{k}: \subseteq \mathbb{N} \rightarrow \mathbb{N}$. If $\left\{F_{k}\right\}_{k \in \mathbb{N}}$ is uniformly computable, then $f$ is computable.

Proof. We define a computable function $F: \subseteq \mathbb{N} \rightarrow \mathbb{N}$ that induces $f$. Let $n \in \mathbb{N}$. Compute the least value of $k$ such that $\bar{R}_{n} \subseteq \overline{\mathbb{D}}_{k}$. If there is no such $k$, then $F(n)$ is undefined. At this point, by using the uniform computability of $\left\{F_{k}\right\}_{k \in N}$, we can proceed as in the proof of the second part of Theorem 2.7.

We will need the following Theorem, which is a consequence of Theorem 4.3.8 of $[13]$.

Theorem 4.4. Let $D$ be a rational disk centered at the origin, and let $\left\{f_{k}\right\}_{k=0}^{\infty}$ be a sequence of functions from $D$ into $\mathbb{C}$ that uniformly converges to $f: D \rightarrow \mathbb{C}$. Suppose there is a uniformly computable family $\left\{F_{k}\right\}_{k \in \mathbb{N}}$ such that for every $k, f_{k}$ is induced by $F_{k}$. Suppose further that there exists an algorithm that given $m \in \mathbb{N}$ as input computes $N$ such that $\left|f_{n}(z)-f_{n^{\prime}}(z)\right|<\frac{1}{2^{k}}$ for all $z \in D$ and all $n, n^{\prime} \geq N$. Then, $f$ is computable. 
We will also need the following lemma, which is Lemma 15.3 of [10].

Lemma 4.5. If $u_{1}, \ldots, u_{N}$ are complex numbers, and if

$$
p_{N}=\prod_{n=1}^{N}\left(1+u_{n}\right), p_{N}^{*}=\prod_{n=1}^{N}\left(1+\left|u_{n}\right|\right),
$$

then

$$
p_{N}^{*} \leq \exp \left(\left|u_{1}\right|+\ldots+\left|u_{N}\right|\right)
$$

and

$$
\left|p_{N}-1\right| \leq p_{N}^{*}-1 \text {. }
$$

Theorem 4.6. Suppose $A=\left\{a_{n}\right\}_{n=0}^{\infty}$ is a computable sequence of complex numbers in the unit disk and that $\sum_{n=0}^{\infty}\left(1-\left|a_{n}\right|\right)$ is a computable real number. Then, $B_{A, m}$ is computable for all $m$.

Proof. Without loss of generality, suppose $m=0$. We apply Lemma 4.3. Fix $k$, and let

$$
\begin{gathered}
r_{k}=1-\frac{1}{2^{k+1}}, \\
C_{1, k}=\frac{1+r_{k}}{1-r_{k}} .
\end{gathered}
$$

Thus, for all $z \in \mathbb{D}_{k}$,

$$
\frac{1+|z|}{1-|z|} \leq C_{1, k}
$$

Furthermore, for all $z \in \mathbb{D}$,

$$
\left|b_{a_{n}}(z)\right| \leq\left|\frac{a_{n}-z}{1-\overline{a_{n}} z}\right| .
$$

Since $z \mapsto \frac{a_{n}-z}{1-\overline{a_{n}} z}$ maps $\mathbb{D}$ into $\mathbb{D},\left|b_{a_{n}}(z)\right| \leq 1$ for all $z \in \mathbb{D}$.

Set $p_{N}=\prod_{n=0}^{N} b_{a_{n}}$. By Theorem 4.4, it suffices to show that we can compute from $k, m$ an $N_{0} \in \mathbb{N}$ such that on $\mathbb{D}_{k},\left|p_{M}-p_{N}\right| \leq \frac{1}{2^{m+1}}$ for all $M, N \geq N_{0}$.

Set $u_{n}=1-b_{a_{n}}$. It follows that

$$
\begin{aligned}
u_{n}(z) & =1-\frac{\left|a_{n}\right|}{a_{n}} \frac{a_{n}-z}{1-\overline{a_{n}} z} \\
& =\frac{a_{n}\left(1-\overline{a_{n}} z\right)-\left|a_{n}\right|\left(a_{n}-z\right)}{a_{n}\left(1-\overline{a_{n}} z\right)} \\
& =\frac{a_{n}-\left|a_{n}\right|^{2} z-\left|a_{n}\right| a_{n}+\left|a_{n}\right| z}{a_{n}\left(1-\overline{a_{n}} z\right)} \\
& =\frac{\left(1-\left|a_{n}\right|\right)\left(a_{n}+\left|a_{n}\right| z\right)}{a_{n}\left(1-\overline{a_{n}} z\right)}
\end{aligned}
$$

It then follows that

$$
\left|u_{n}(z)\right| \leq \frac{1+|z|}{1-|z|}\left(1-\left|a_{n}\right|\right) \leq C_{1, k}\left(1-\left|a_{n}\right|\right)
$$

for all $z \in \mathbb{D}_{k}$. Since $\left\{a_{n}\right\}_{n=0}^{\infty}$ and $\sum_{n=0}^{\infty}\left(1-\left|a_{n}\right|\right)$ are computable, we can compute a function $f: \mathbb{N} \rightarrow \mathbb{N}$ such that for all $m$

$$
\sum_{n=f(m)}^{\infty}\left(1-\left|a_{n}\right|\right)<\frac{1}{2^{m} C_{1, k}} .
$$


Let $N_{0}=f(m+1)$. Set $\epsilon=\frac{1}{2^{m+2}}$. Suppose $M>N \geq N_{0}$. It follows from Lemma 4.5 that on $\mathbb{D}_{k}$

$$
\begin{aligned}
\left|p_{M}-p_{N}\right| & =\left|p_{N}\right|\left|\frac{p_{M}}{p_{N}}-1\right| \\
& \leq\left|p_{N}\right|\left(\exp \left(\left|u_{N+1}\right|+\ldots+\left|u_{M}\right|\right)-1\right) \\
& \leq\left|p_{N}\right|\left(e^{\epsilon}-1\right) \leq 2\left|p_{N}\right| \epsilon \leq 2\left|p_{1}\right| \epsilon \leq 2 \epsilon=\frac{1}{2^{m+1}}
\end{aligned}
$$

We do not know if the computability of a Blaschke sum is equivalent to the computability of the corresponding Blaschke product.

Corollary 4.7. Suppose $A=\left\{a_{n}\right\}_{n=0}^{\infty}$ is a computable sequence of complex numbers in the unit disk and that $\lim \sup \frac{1-\left|a_{n+1}\right|}{1-\left|a_{n}\right|}<1$. Then, $B_{A, m}$ is computable for all $m$.

Proof. There is a rational $R \in(0,1)$ and an integer $t$ such that

$$
\frac{1-\left|a_{n+1}\right|}{1-\left|a_{n}\right|} \leq R
$$

for all $n \geq t$. It follows that $1-\left|a_{t+j}\right| \leq R^{j}\left(1-\left|a_{t}\right|\right)$ for all $j \geq 0$. Hence, for all $k \geq t$

$$
\sum_{n=k}^{\infty}\left(1-\left|a_{n}\right|\right) \leq\left(1-\left|a_{t}\right|\right) \sum_{n=k-t}^{\infty} R^{n} \leq \frac{R^{k-t}}{1-R} .
$$

It then follows that $\sum_{n=0}^{\infty}\left(1-\left|a_{n}\right|\right)$ is a computable real number.

The following proposition shows that the converse of Corollary 4.7 does not hold.

Proposition 4.8. There exists a computable sequence of real numbers in the unit disk, $A=\left\{a_{n}\right\}_{n=0}^{\infty}$, such that $B_{A}$ is computable and $\lim \sup \frac{1-\left|a_{n+1}\right|}{1-\left|a_{n}\right|}=1$.

Proof. Let $a_{n}=1-\frac{1}{(n+1)^{2}}$. We note that

$$
\sum_{n=k}^{\infty} \frac{1}{(n+1)^{2}} \leq \int_{k}^{\infty} \frac{1}{x^{2}} d x=\frac{1}{k} .
$$

It then follows that $\sum_{n=0}^{\infty}\left(1-\left|a_{n}\right|\right)$ is computable.

Definition 4.9. A sequence $A=\left\{a_{n}\right\}_{n=0}^{\infty}$ of points in $\mathbb{D}$ is interpolating if for every bounded sequence of points in $\mathbb{C},\left\{z_{n}\right\}_{n=0}^{\infty}$, there exists a bounded analytic function $f: \mathbb{D} \rightarrow \mathbb{C}$ such that $f\left(a_{n}\right)=z_{n}$ for all $n$.

A Blaschke product is called interpolating if it has an interpolating sequence of zeros. In [3], it is shown that every inner function can be approximated by a linear combination of interpolating Blaschke products. It is not known whether the interpolating Blaschke products are uniformly dense in the space of inner functions.

Definition 4.10. A sequence $A=\left\{a_{n}\right\}_{n=0}^{\infty}$ of points in $\mathbb{D}$ is radial if its points all lie on a single radius of $\mathbb{D}$.

It is well-known that a radial sequence $\left\{a_{n}\right\}_{n=0}^{\infty}$ is interpolating if and only if $\lim \sup \frac{1-\left|a_{n+1}\right|}{1-\left|a_{n}\right|}<1$. We thus obtain the following.

Corollary 4.11. If $A$ is computable, interpolating, and radial, then $B_{A, m}$ is computable for all $m$. 
We do not know if there is a computable interpolating sequence that defines a non-computable Blaschke product.

\section{Concluding REmarks}

We note that the results in this paper are similar to certain results on power series. First, there is the result that, roughly speaking, one can compute the power series expansion of an analytic function from the function itself. A precise statement and proof of this result can be found in Theorem 6.5.2 of [13]. We also note the following surprising result of Caldwell and Pour-El [2].

Theorem 5.1. There is an analytic function $f: \mathbb{C} \rightarrow \mathbb{C}$ such that $f$ is not computable, but its restriction to any compact disk is computable.

We do not know if there is a formal relation between these results on power series and our results on Blaschke products.

\section{ACKNOWLEGMENTS}

We thank the referee for his many helpful comments. In particular, the referee's remarks led to significant improvements in the proofs of Theorems 2.7 and 3.4. We also thank Iraj Kalantari, Charles Coppin, Valentin Andreev, and Susan McNicholl.

\section{REFERENCES}

1. J. Bak, R. J. Newman, Complex Analysis, 1st ed. (Springer-Verlag, New York, 1982). MR671250 (84b:30001)

2. J. Caldwell, M. B. Pour-El. On a simple definition of computable functions of a real variablewith applications to functions of a complex variable. Zeitschrift für Mathematische Logik und Grundlagen der Mathematik, vol. 21 (1975), pp. 1 - 19. MR0366638 (51:2885)

3. J. Garnett, Interpolating Blaschke products generate $H^{\infty}$. Pacific Journal of Mathematics, vol. 173 (1996), pp. 501 - 510. MR1394402 (97f:30050)

4. J. Garnett, Bounded Analytic Functions, 1st ed. (Academic Press, 1981). MR628971 (83g:30037)

5. P. Hertling, An effective Riemann Mapping Theorem. Theoretical Computer Science, vol. 219 (1999), pp. 225 - 265. MR1694433 (2000f:03187)

6. G. A. Hjelle, Unimodular functions and interpolating Blaschke products. Proceedings of the American Mathematical Society, vol. 134 (2006), pp. 207 - 214. MR2170560 (2006f:30039)

7. I. Kalantari and L. Welch, Recursive and nonextendible functions over the reals; filter foundation for recursive analysis. II. Annals of Pure and Applied Logic, vol. 98 (1999), no. 1 - 3, pp. 87 - 110. MR1696843 (2000g:03098)

8. I. Kalantari and L. Welch, Specker's theorem, cluster points, and computable quantum functions, Logic in Tehran, Lecture Notes in Logic, vol. 26 (Association for Symbolic Logic, 2006). MR2262318

9. A. Matheson and M. Stessin, Cauchy Transforms of characteristic functions and algebras generated by inner functions. Proceedings of the American Mathematical Society, vol. 133 (2005), pp. 3361 - 3370. MR2161161 (2006f:30040)

10. W. Rudin, Real and Complex Analysis, 3rd ed. (McGraw-Hill, 1987). MR924157 (88k:00002)

11. R. I. Soare, Recursively Enumerable Sets and Degrees, 1st ed. (Springer-Verlag, Berlin, Heidelberg, 1987). MR882921 (88m:03003)

12. E. Specker, Nicht konstruktiv beweisbare Sätze der Analysis. The Journal of Symbolic Logic, vol. 14 (1949), pp. 145 - 158. MR0031447 (11:151g)

13. K. Weihrauch, Computable Analysis. An Introduction, 1st ed. (Springer-Verlag, Berlin, 2000). MR1795407 (2002b:03129)

Department of Mathematics, Lamar University, Beaumont, Texas 77710

Department of Mathematics, Lamar University, Beaumont, Texas 77710

E-mail address: mcnicholl@math.lamar.edu 